\title{
Neovascularization and oxidative stress in the progression of non-alcoholic steatohepatitis
}

\author{
MITSUTERU KITADE, HITOSHI YOSHIJI, HIDEYUKI KOJIMA, YASUHIDE IKENAKA, RYUICHI NOGUCHI, \\ KOSUKE KAJI, JUNICHI YOSHII, KOJI YANASE, TADASHI NAMISAKI, MASAHARU YAMAZAKI, \\ TATSUHIRO TSUJIMOTO, KEI MORIYA, HIDETO KAWARATANI, \\ TAKEMI AKAHANE and HIROSHI FUKUI
}

Third Department of Internal Medicine, Nara Medical University, Kashihara, Nara, Japan

Received February 29, 2008; Accepted April 29, 2008

\begin{abstract}
Reactive oxygen species (ROS) is known to play an important role in the pathogenesis of non-alcoholic steatohepatitis (NASH); however, as we previously reported, angiogenesis also plays a pivotal role in NASH progression the development of liver fibrosis and hepatocellular carcinoma - in rats. The aim of the current study was to elucidate the role of angiogenesis in the development of fibrosis in NASH. Twenty-six patients with NASH and 11 with simple fatty liver (FL) disease were enrolled in the study and underwent clinicopathological examination. Immunohistochemical analysis of 4-hydroxy-2-noneal (4-HNE) and CD34 was employed for the detection of ROS and angiogenesis in the liver tissues, respectively. Both the NASH and FL samples displayed a marked staining of 4-HNE as compared to the healthy liver. Similar levels of 4-HNE were observed in NASH regardless of the grade of liver fibrosis. In sharp contrast, hepatic neovascularization developed significantly in NASH alone, whereas almost no neovascularization was observed in FL or the healthy liver. The degree of angiogenesis was almost parallel with the development of liver fibrosis. In conclusion, simple FL and NASH cases were both affected by ROS. However, only NASH was associated with marked neovascularization, proportional to the increase in the grade of liver fibrosis development. These results indicate that hepatic neovascularization may play an important role in the onset and progression of NASH.
\end{abstract}

Correspondence to: Dr Hitoshi Yoshiji, Third Department of Internal Medicine, Nara Medical University, Shijo-cho 840, Kashihara, Nara 634-8522, Japan

E-mail: yoshijih@naramed-u.ac.jp

Abbreviations: AST, aspartate aminotransferase; BMI, body mass index; ECs, endothelial cells; FL, fatty liver; HSCs, hepatic stellate cells; NAFLD, non-alcoholic fatty liver disease; NASH, non-alcoholic steatohepatitis; ROS, reactive oxygen species; VEGF, vascular endothelial growth factor; 4-HNE, 4-hydroxy-2-noneal; 8-OHdG, 8-hydroxydeoxyguanosine

Key words: non-alcoholic steatohepatitis, liver fibrosis, angiogenesis, oxidative stress

\section{Introduction}

Non-alcoholic fatty liver disease (NAFLD) is one of the major consequences of the current obesity epidemic $(1,2)$, and ranges from simple steatosis to cirrhosis. Fatty liver (FL) disease has been known as a benign and non-progressive condition. However, non-alcoholic steatohepatitis (NASH) is now widely recognized as a serious liver disease which may progress to liver cirrhosis and, finally, to hepatocellular carcinoma. The pathogenesis of NASH is reportedly a multi-factorial process (2). Recently, the focus has been on reactive oxygen species (ROS), a common pathogenic mechanism in various liver diseases, including NASH $(3,4)$. It was found that hepatic 4-hydroxy-2-noneal (4-HNE) and 8-hydroxydeoxyguanosine (8-OHdG), as reliable ROS markers of lipid peroxidation and oxidative DNA damage, respectively, were significantly increased in animal models and human samples of NASH $(5,6)$. However, it has not yet been elucidated whether ROS is a crucial factor in the progression of NASH since, as in diabetes mellitus, NASH is almost certainly a polygenic disease affected by several factors related to 'multiple hits' (2).

Angiogenesis, the formation of new vessels which sprout from pre-existing vasculature, is of central importance to embryonic development and organogenesis. In adults, physiological angiogenesis occurs during the female reproductive cycle and in wound healing (7). Abnormal pathological angiogenesis is observed in rheumatoid arthritis, psoriasis, diabetic retinopathy, tumor growth and even in fibrogenesis (7). Although early studies conducted to determine the molecular processes associated with fibrosis and angiogenesis were performed independently, more recent studies have revealed that both biological phenomena emerge synergistically (8). It has been shown that neovascularization significantly increases during the development of liver fibrosis in both human and animal experimental studies $(9,10)$. Among the angiogenic factors identified to date, vascular endothelial growth factor (VEGF) is one of the most potent and central in many physiological and pathological processes (11). In liver fibrosis, VEGF expression is increased in both human chronic liver diseases and in experimental fibrogenesis $(10,12,13)$. VEGF expression reportedly correlates with chronic liver diseaseassociated angiogenesis and sinusoidal capillarization $(12,14)$. These results suggest that VEGF-mediated angiogenesis also plays an important role in the development of liver fibrosis. In 
the NASH experimental model, we previously demonstrated that angiogenesis, which coordinates with VEGF, plays an important role in the progression of NASH (15). However, as of yet no study has been conducted to examine the role of angiogenesis in NASH with human samples.

In the current study, we examined the possible involvement of angiogenesis in patients with NAFLD, i.e., FL and NASH, particularly in conjunction with ROS. We also elucidated the possible correlation between an increase in hepatic neovascularization and the stage of liver fibrosis development.

\section{Patients and methods}

Patients and data evaluation. In the current study, a total of 37 patients with NAFLD enrolled between 2001 and 2004 at Nara Medical University were examined. The 37 patients comprised 26 with NASH (15 men and 11 women) and 11 with simple FL (7 men and 4 women) diagnosed by histological examination. Three healthy volunteers were enrolled as a control group. First, all patients were re-evaluated clinically for evidence of diseases including diabetes mellitus and hypertension. Alcoholinduced hepatitis was excluded according to a patient selfreport confirmed by the family. Patient height and weight were measured, and the body mass index (BMI) was calculated. Hepatitis B virus surface antigen and hepatitis $C$ virus antibody were negative in all patients. Standard liver function tests were performed for all patients. Fasting blood levels of glucose and insulin were determined and the homeostasis model assessment parameter of insulin resistance (HOMA-IR) was calculated. The serum fibrosis markers hyaluronic acid, type IV collagen $7 \mathrm{~S}$ and amino-terminal peptide of type-III pro-collagen were measured before and after treatment in all patients by latex agglutination, enzyme immunoassay and radioimmunoassay, respectively, using routine laboratory methods. The serum levels of leptin and adiponectin were measured using the ELISA kit (R\&D Systems, Tokyo, Japan) according to the manufacturer's instructions as described previously $(16,17)$.

Histology and immunohistochemistry. Liver biopsies obtained for diagnostic purposes were histologically examined. In all samples, serial sections were used for analysis. The first section was routinely stained with hematoxylin and eosin for histological examination. Another section was stained with Sirius-red to detect the stage of fibrosis development, which was scored on a scale of F0 to F4: F0, the absence of fibrosis; F1, portal fibrosis with few septa; F2, few septa; F3, abundant septa with cirrhosis; F4, cirrhosis as described elsewhere (18). Of the 26 patients with NASH, 12 had low-grade (F0, F1) and 14 high-grade fibrosis (F2-F4). In order to determine the extent of neovascularization, immunohistochemical detection of CD34, which is widely used as a marker of neovascularization, was employed using paraffin-embedded sections as described previously (19). Immunohistochemical staining of 4-HNE as a marker of ROS-induced lipid peroxidation in the liver was also performed as described previously (5). Liver tissue samples were fixed in $4 \%$ formaldehyde solution and embedded in paraffin. Then, $5-\mu \mathrm{m}$ tissue sections were incubated in $0.1 \%$ hydrogen peroxide in $70 \%$ methanol for $30 \mathrm{~min}$ to inhibit endogenous peroxidase activity. Micro-wave antigen retrieval was performed at $500 \mathrm{~W}$ for $15 \mathrm{~min}$ with antigen retrieval solution, pH 9.0 (Nichirei Bioscience Inc., Tokyo, Japan). Then, $10 \%$ fetal bovine serum with $0.3 \%$ Triton-X was used to prevent non-specific staining. The slides were subsequently incubated overnight at $4^{\circ} \mathrm{C}$ in humidified chambers with primary rabbit polyclonal anti-CD34 antibody at a dilution of 1:100 and primary rabbit polyclonal anti-4-HNE antibody (NOF Corp., Tokyo, Japan) at a dilution of 1:100, respectively. The sections were then rinsed three times in a phosphate-buffered solution and further incubated with a biotinylated secondary antibody for $30 \mathrm{~min}$ at room temperature. Antigen-antibody complexes were detected by the avidin-biotin-peroxide method using diaminobenzidine as a chromogenic substrate (Dako, Carpinteria, CA, USA). Finally, the slides were counterstained with hematoxylin then examined microscopically. Immunopositive quantitation of CD34-, 4-HNE- and Sirius-red-positive liver fibrotic areas were evaluated with Adobe Photoshop and NIH Image software as described previously (20).

Statistical analysis. All data were expressed as the means \pm SD. Statistical analysis was performed using the $\chi^{2}$ test for independence, the 2-tailed Student's t-test and simple regression analysis.

\section{Results}

Clinical findings. The characteristic features of both groups are shown in Table I. Most of the clinical features of the patients with FL and NASH were not significantly different, with the exception of the BMI and serum aspartate aminotransferase (AST), hyaluronic acid, leptin and adiponectin levels. Patients with NASH had a high BMI $(28.8 \pm 4.33$ vs. $25.6 \pm 1.20)$, AST $(59.4 \pm 27.0$ vs. $37.5 \pm 10.0)$, hyaluronic acid $(62.0 \pm 58.6$ vs. $15.0 \pm 9.06)$ and leptin $(7.79 \pm 4.47$ vs. $2.58 \pm 0.43)$ as compared to FL patients. On the other hand, a lower serum adiponectin level was observed in NASH vs. FL (5.31 \pm 1.70 vs. $8.25 \pm 0.95)$ patients.

ROS expression in NAFLD. ROS-induced lipid peroxidation was estimated by the prevalence and intensity of 4-HNE. Staining for 4-HNE was barely visible in the healthy control liver (Fig. 1A), whereas most cases of both FL and NASH showed diffuse granular staining (Fig. 1B and C, respectively). Semi-quantitative analysis showed that ROS-induced lipid peroxidation was more advanced both in FL and NASH than in the healthy control liver (Fig. 2). However, there was no significant difference between FL and NASH in the 4-HNE staining index. Furthermore, a similar 4-HNE index was observed in low- (F0, F1) and high- (F2-F4) grade fibrosis in NASH patients (Fig. 2). The results of the 8-OHdG-positive index were similar to those of 4-HNE (data not shown).

Neovascularization in NAFLD. The possible interaction between the progression of NASH and hepatic neovascularization was examined next, as previous studies have revealed that angiogenesis plays an important role in liver fibrosis development $(9,10)$. The typical features of CD34-immunopositive neovessels in the liver are shown in Fig. 3. In sharp contrast to the 4-HNE findings, a significant difference was observed between FL and NASH cases. Apparent CD34- 
Table I. Characteristic features of patients with FL and NASH.

\begin{tabular}{|c|c|c|c|}
\hline & NASH $(n=26)$ & $\mathrm{FL}(\mathrm{n}=11)$ & P-value \\
\hline Age (years) & $46.5 \pm 15.0$ & $40.1 \pm 11.7$ & 0.214 \\
\hline Sex (male/female) & $15 / 11$ & $7 / 4$ & 0.595 \\
\hline BMI $\left(\mathrm{kg} / \mathrm{m}^{2}\right)$ & $28.8 \pm 4.33$ & $25.6 \pm 1.20$ & 0.022 \\
\hline Total cholesterol (mg/dl) & $222.0 \pm 43.3$ & $203.4 \pm 48.3$ & 0.254 \\
\hline Triglycerides (mg/dl) & $190.0 \pm 75.9$ & $237.1 \pm 98.2$ & 0.123 \\
\hline FFA (mmol/l) & $0.528 \pm 0.220$ & $0.897 \pm 0.804$ & 0.096 \\
\hline Fasting plasma glucose (mg/dl) & $109.7 \pm 30.2$ & $96.3 \pm 14.8$ & 0.219 \\
\hline Fasting plasma insulin $(\mu \mathrm{U} / \mathrm{ml})$ & $18.2 \pm 12.0$ & $13.3 \pm 4.75$ & 0.280 \\
\hline HOMA-IR & $5.34 \pm 4.68$ & $3.16 \pm 0.96$ & 0.210 \\
\hline HbA1c $(\%)$ & $5.85 \pm 1.15$ & $5.25 \pm 0.53$ & 0.110 \\
\hline Serum albumin (g/dl) & $4.56 \pm 0.37$ & $4.54 \pm 0.39$ & 0.876 \\
\hline Aspartate aminotransferase (IU/l) & $59.4 \pm 27.0$ & $37.5 \pm 10.0$ & 0.014 \\
\hline Alanine aminotransferase (IU/l) & $93.4 \pm 45.7$ & $69.8 \pm 31.2$ & 0.135 \\
\hline$\gamma$-GTP (IU/l) & $70.3 \pm 38.0$ & $64.2 \pm 47.8$ & 0.683 \\
\hline ZTT (KU) & $8.18 \pm 4.62$ & $5.89 \pm 2.39$ & 0.166 \\
\hline Total bilirubin (mg/dl) & $0.81 \pm 0.26$ & $1.00 \pm 0.98$ & 0.333 \\
\hline ICG R15' (\%) & $10.8 \pm 4.51$ & $9.00 \pm 2.61$ & 0.310 \\
\hline Procollagen-III-peptide (U/ml) & $0.85 \pm 0.32$ & $0.96 \pm 0.23$ & 0.501 \\
\hline Collagen IV-7S (ng/ml) & $4.40 \pm 1.86$ & $4.40 \pm 1.52$ & 0.997 \\
\hline Hyaluronic acid (ng/ml) & $62.0 \pm 58.6$ & $15.0 \pm 9.06$ & 0.034 \\
\hline Platelets $\left(10^{4} / \mu 1\right)$ & $20.9 \pm 8.83$ & $23.2 \pm 4.85$ & 0.424 \\
\hline Leptin (ng/ml) & $7.79 \pm 4.47$ & $2.58 \pm 0.43$ & 0.036 \\
\hline Adiponectin (ng/ml) & $5.31 \pm 1.70$ & $8.25 \pm 0.95$ & 0.005 \\
\hline
\end{tabular}
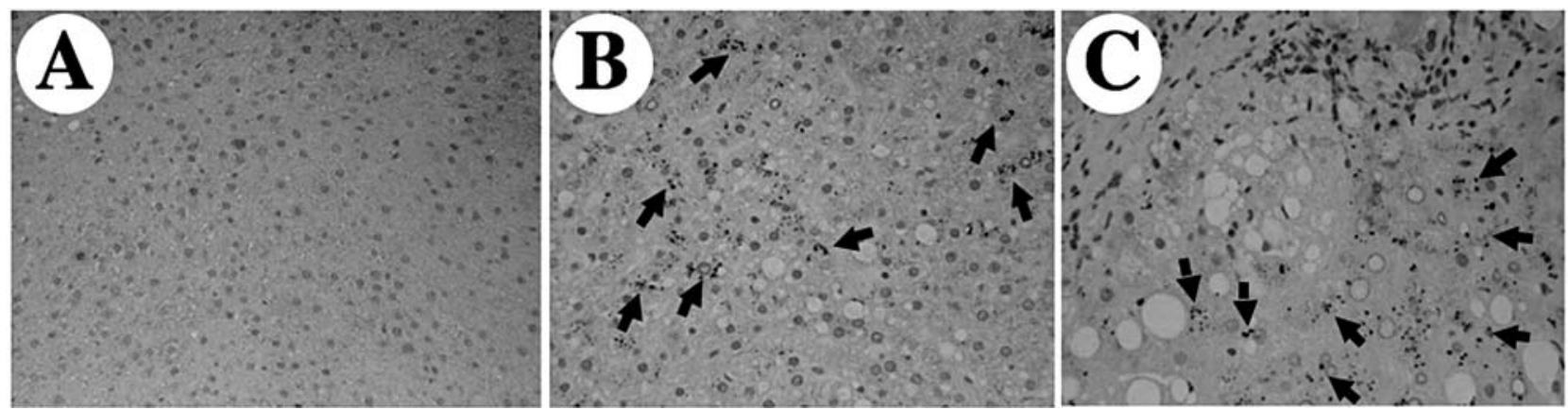

Figure 1. Microphotographs of 4-HNE-positive ROS production in the liver of patients with NAFLD. ROS-induced lipid peroxidation was measured by 4-HNE immunohistochemical analysis. Staining of 4-HNE was barely visible in the healthy control liver (A), whereas most cases of both FL and NASH showed diffuse granular staining (B and C, respectively). There was no significant difference in 4-HNE staining between FL and NASH samples. Arrows indicate 4-HNEimmunopositivity. Original magnification, $\mathrm{x} 400$.

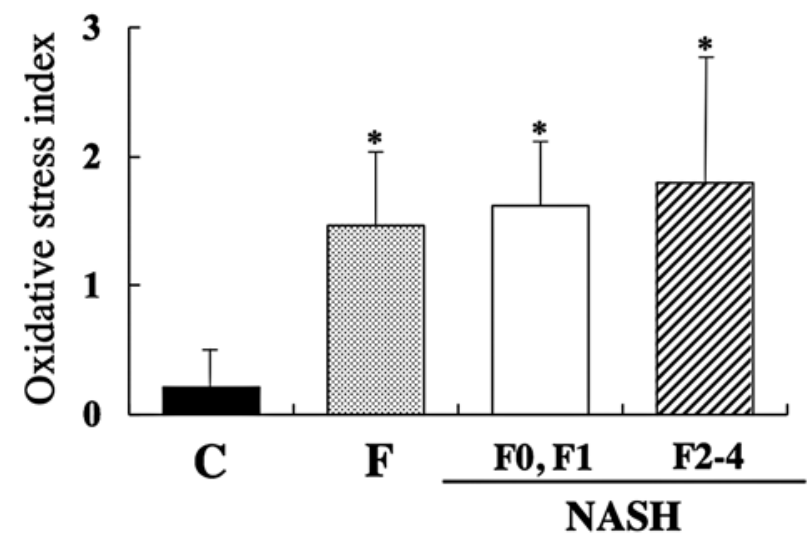

Figure 2. Semi-quantitative analysis of ROS production in the NAFLD liver. Both FL and NASH cases showed a marked increase in the hepatic 4-HNE index as compared to the healthy control liver, and there was no significant difference between FL and NASH samples. In NASH, a similar 4-HNE-index was observed in low- (F0, F1) and high- (F2-F4) grade fibrosis development. $\mathrm{C}$, healthy control group ( $\mathrm{n}=3) ; \mathrm{F}$, FL group ( $\mathrm{n}=11) ; \mathrm{F} 0, \mathrm{~F} 1$, low-grade fibrosis $(\mathrm{n}=12)$; F2-4, high-grade fibrosis ( $\mathrm{n}=14)$; NASH, NASH group. *Statistically significant difference as compared to the control group $(\mathrm{p}<0.01)$. 

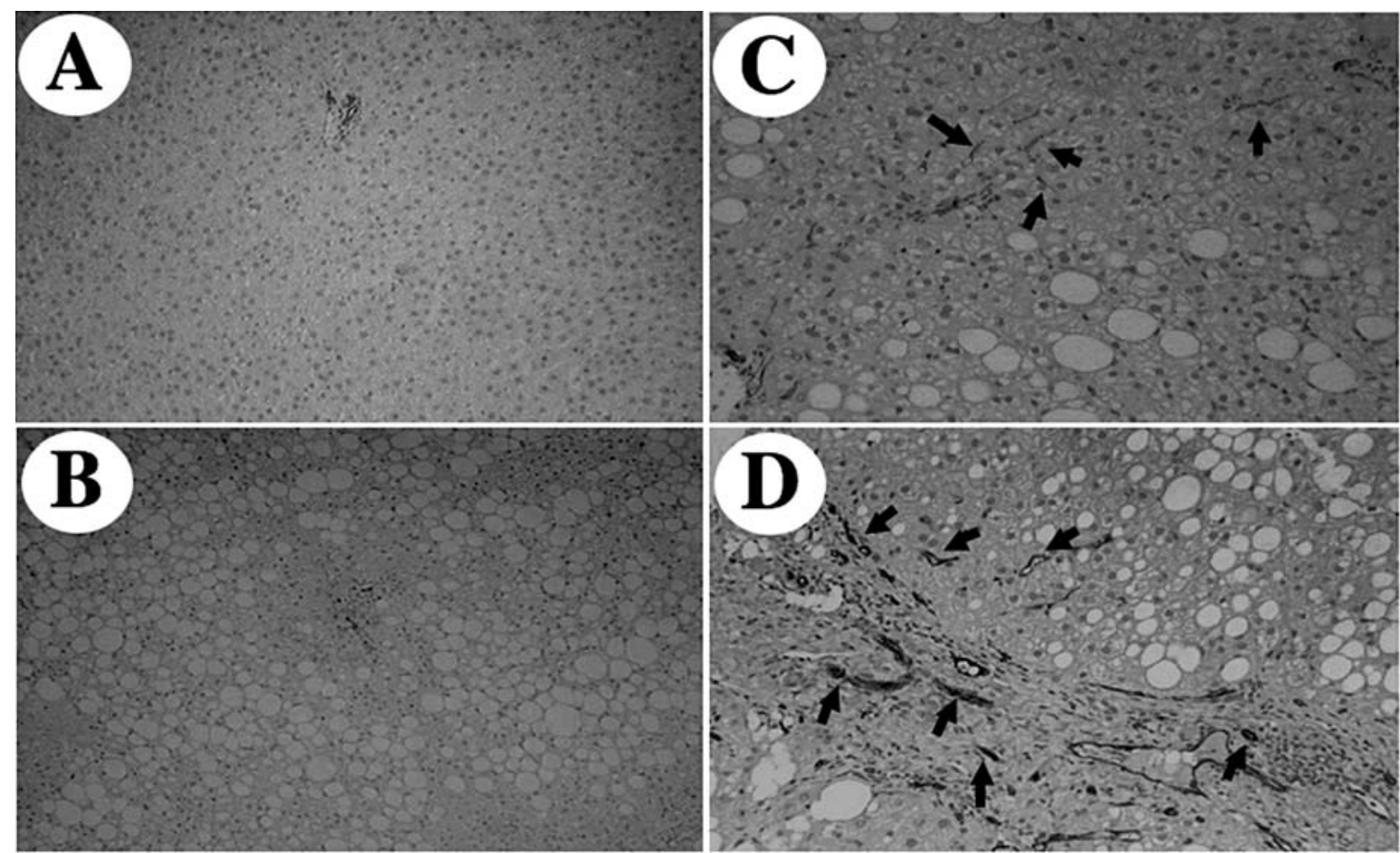

Figure 3. Microphotographs of CD34-positive neovessels in the liver of patients with NAFLD. In sharp contrast to 4-HNE, a significant difference was observed between the FL and NASH cases. CD34-positive vessels were not apparent in either the healthy control liver or FL (A and B, respectively), whereas marked CD34-immunopositive vessels were observed in NASH along with the development of liver fibrosis. In the low-grade fibrosis liver (F1), neovascularization was observed around the central vein (zone-III) (C). These neovessels progressed to the portal area (zone-I) and were also observed along the fibrotic septa in the highgrade fibrosis liver (F3) (D). Arrows indicate CD34-positive neovessels. Original magnification, x100.

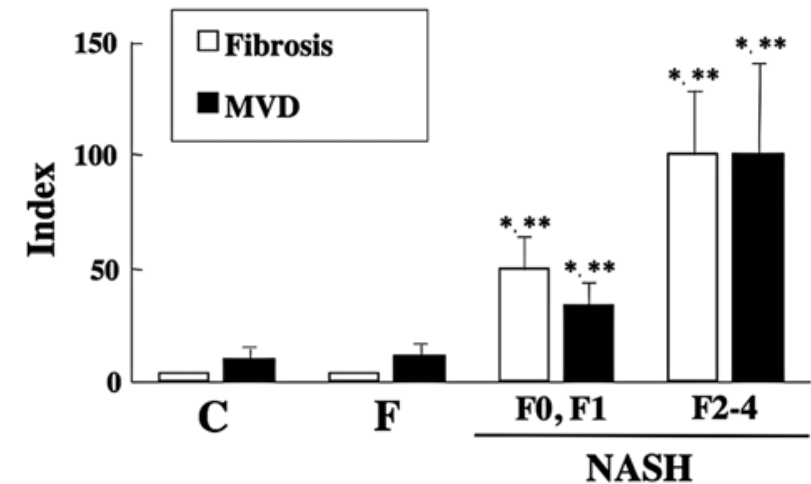

Figure 4. Semi-quantitative analysis of CD34-positive neovessels in the NAFLD liver. There was no difference between the healthy control liver and FL cases. In NASH, a marked augmentation of neovascularization was found as compared to FL. The magnitude of neovascularization in high-grade (F2-F4) liver fibrosis development was greater than in the low-grade (F0, F1) fibrosis. The proportional increment of neovascularization was almost parallel with the development of liver fibrosis. C, healthy control group (n=3); F, FL group ( $\mathrm{n}=11)$; F0, F1, low-grade fibrosis $(\mathrm{n}=12)$; F2-4, high-grade fibrosis $(\mathrm{n}=14)$; NASH, NASH group; MVD, microvessel density. ${ }^{*, * *}$ Statistically significant differences between the control group and the low-grade fibrosis and NASH groups, respectively $(\mathrm{p}<0.01)$.

positive vessels were observed neither in the liver of the healthy subjects nor in FL patients (Fig. 3A and B, respectively), whereas marked immunopositive vessels were observed in NASH along with liver fibrosis development (Fig. 3C and D). In the liver of low-grade fibrosis, neovascularization was observed around the central vein (zone-III) (Fig. 3C). These neovessels progressed to the portal area (zone-I) and were also observed along the fibrotic septa in high-grade fibrosis (Fig. 3D). Next, a semi-quantitative analysis of the CD34positive neovessels in conjunction with liver fibrosis development was performed. There was no difference between the normal control liver and FL (Fig. 4), which conformed with the immunohistochemical features. In NASH, a marked augmentation of neovascularization was found when controls were compared with FL samples. The magnitude of neovascularization in high-grade liver fibrosis development was higher than in low-grade fibrosis. The increase in neovascularization was almost parallel with the development of liver fibrosis.

\section{Discussion}

Several studies have revealed that the pathogenesis of NASH most likely involves multiple steps, and it is widely recognized to be explained by the 'two-hit theory'. The second hit, which occurs sequentially after the first, is based on several metabolic disorders such as insulin resistance and leads to the progression of NASH. Several studies have suggested that ROS is the leading culprit behind the second hit (21). Consequently, the focus as been trained on ROS, a common pathogenic mechanism in various liver diseases including NAFLD $(3,4)$. In addition, 4-HNE and $8-\mathrm{OHdG}$ have been reported to be over-expressed in NASH livers (5). However, another study found that ROS was significantly increased in FL as well as NASH livers (22), indicating that FL and NASH are both affected by ROS despite the different clinical outcomes. It has been reported that increased production of ROS occurs very early in the histological spectrum of NAFLD (6), and has also been demonstrated that, even at the earliest stage of 
NAFLD, considerable oxidative stress already exists (23). Taken together, it is feasible that the onset and progression of NASH can be initiated by the introduction of an additional factor based on the augmentation of ROS.

Among the possible factors involved, we focused on neovascularization. It is now widely recognized that angiogenesis plays an important role in many pathological events, including liver fibrosis development. Angiogenesis in the liver is characterized by capillarization of the sinusoids (7). It has been shown that capillarization and phenotypic changes of the hepatic sinusoidal endothelial cells (ECs) occur during liver fibrosis development (24), and has been reported that CD34 is not expressed by healthy ECs. Rather, when ECs alter their phenotype, they can express CD34 (25). In the current study, we found a significant development of hepatic CD34-positive neovascularization in NASH, whereas almost no development was observed in FL. The degree of angiogenesis was almost parallel with that of liver fibrosis in NASH. These results are consistent with our previous finding that angiogenesis increases stepwise during hepatic fibrosis development in several models of fibrosis, including the rodent dietary NASH model $(9,15)$. Collectively, and similarly to ROS, angiogenesis plays a pivotal role in the development of fibrosis in NASH.

It was also important to identify the localization of neovascularization during the progression of NASH. CD34-positive neovessels were initially observed around the central vein (zone-III), then gradually progressed to the portal area (zone-I). They were also observed along the fibrotic septa in highgrade fibrosis. These progression patterns are similar to those of fibrosis development in NASH $(1,2)$, and it is likely that neovascularization in NASH progresses almost concurrently with fibrosis development. Further studies are required to elucidate its exact mechanisms.

Since NAFLD is a common manifestation of metabolic syndromes, various adipocytokines are involved in its progression $(26,27)$. Among these are adiponectin and leptin, swhich are known to be involved in the pathogenesis of NASH $(26,28,29)$. Adiponectin administration alleviates NAFLD progression in mice, and liver fibrosis is accelerated in adiponectin knockout mice. This indicates that adiponectin has a protective effect against liver fibrosis development in NASH (30). In NASH patients, circulating adiponectin reportedly decreased (29), and serum levels of adiponectin significantly decreased in NASH compared to FL patients. On the other hand, previous studies have revealed that leptin exerts a pro-fibrogenic activity, and that hepatic fibrogenesis is impaired in leptin- and leptin receptor-deficient animals $(28,31)$. Leptin also enhances the proliferation of activated hepatic stellate cells (HSCs), which play a central role in liver fibrosis development (32). In addition to these direct effects on HSCs, studies have revealed that leptin possesses angiogenic activity $(15,33)$. We previously reported that leptin exerts potent angiogenic activity coordinated with VEGF, and that leptin-mediated neovascularization plays an important role in the development of liver fibrosis in the rat NASH model (15). In the current study, the leptin serum level was significantly higher in NASH compared to FL patients. These results suggest that, as in the animal model, leptin-mediated angiogenesis plays some role in the progression of NASH in humans.
In conclusion, the present study revealed that both FL and NASH are greatly affected by ROS. However, a marked development of hepatic neovascularization was observed only in NASH, with almost no development in FL. The degree of angiogenesis was almost parallel with liver fibrosis development, indicating that hepatic neovascularization plays an important role in the onset and progression of NASH. Thus, anti-angiogenic therapy may become an alternative new strategy against NASH.

\section{References}

1. Angulo P: Nonalcoholic fatty liver disease. N Engl J Med 346: 1221-1231, 2002.

2. Reid AE: Nonalcoholic steatohepatitis. Gastroenterology 121: 710-723, 2001.

3. Jaeschke H: Reactive oxygen and mechanisms of inflammatory liver injury. J Gastroenterol Hepatol 15: 718-724, 2000.

4. Sato N: Central role of mitochondria in metabolic regulation of liver pathophysiology. J Gastroenterol Hepatol 22 (suppl 1): 1-6, 2007.

5. Seki S, Kitada T, Yamada T, Sakaguchi H, Nakatani K and Wakasa K: In situ detection of lipid peroxidation and oxidative DNA damage in non-alcoholic fatty liver diseases. J Hepatol 37 : 56-62, 2002

6. Gao D, Wei C, Chen L, Huang J, Yang S and Diehl AM: Oxidative DNA damage and DNA repair enzyme expression are inversely related in murine models of fatty liver disease. Am J Physiol Gastrointest Liver Physiol 287: G1070-G1077, 2004.

7. Carmeliet P: Angiogenesis in life, disease and medicine. Nature 438: 932-936, 2005.

8. Kalluri R and Sukhatme VP: Fibrosis and angiogenesis. Curr Opin Nephrol Hypertens 9: 413-418, 2000.

9. Yoshiji H, Kuriyama S, Yoshii J, et al: Vascular endothelial growth factor and receptor interaction is a prerequisite for murine hepatic fibrogenesis. Gut 52: 1347-1354, 2003.

10. Medina J, Caveda L, Sanz-Cameno P, et al: Hepatocyte growth factor activates endothelial proangiogenic mechanisms relevant in chronic hepatitis C-associated neoangiogenesis. J Hepatol 38: 660-667, 2003.

11. Shibuya M: Structure and function of VEGF/VEGF-receptor system involved in angiogenesis. Cell Struct Funct 26: 25-35, 2001.

12. Corpechot C, Barbu V, Wendum D, et al: Hypoxia-induced VEGF and collagen I expressions are associated with angiogenesis and fibrogenesis in experimental cirrhosis. Hepatology 35: 1010-1021, 2002 .

13. Salcedo X, Medina J, Sanz-Cameno P, et al: The potential of angiogenesis soluble markers in chronic hepatitis $\mathrm{C}$. Hepatology 42: 696-701, 2005.

14. Nakamura K, Zen Y, Sato Y, et al: Vascular endothelial growth factor, its receptor Flk-1, and hypoxia inducible factor-1alpha are involved in malignant transformation in dysplastic nodules of the liver. Hum Pathol 38: 1532-1546, 2007.

15. Kitade M, Yoshiji H, Kojima H, et al: Leptin-mediated neovascularization is a prerequisite for progression of nonalcoholic steatohepatitis in rats. Hepatology 44: 983-991, 2006.

16. Zhang Y, Proenca R, Maffei M, Barone M, Leopold L and Friedman JM: Positional cloning of the mouse obese gene and its human homologue. Nature 372: 425-432, 1994.

17. Yamauchi T, Kamon J, Ito Y, et al: Cloning of adiponectin receptors that mediate antidiabetic metabolic effects. Nature 423: 762-769, 2003.

18. The French METAVIR Cooperative Study Group: Intraobserver and interobserver variations in liver biopsy interpretation in patients with chronic hepatitis C. Hepatology 20: 15-20, 1994.

19. Di Carlo I, Fraggetta F, Lombardo R, Azzarello G, Vasquez E and Puleo S: CD 34 expression in chronic and neoplastic liver diseases. Panminerva Med 44: 365-367, 2002.

20. Yoshiji H, Kuriyama S, Yoshii J, et al: Synergistic effect of basic fibroblast growth factor and vascular endothelial growth factor in murine hepatocellular carcinoma. Hepatology 35: 834-842, 2002.

21. Pessayre D, Mansouri A and Fromenty B: Nonalcoholic steatosis and steatohepatitis. V. Mitochondrial dysfunction in steatohepatitis. Am J Physiol Gastrointest Liver Physiol 282: G193-G199, 2002 . 
22. Kojima H, Sakurai S, Uemura M, Fukui H, Morimoto H and Tamagawa Y: Mitochondrial abnormality and oxidative stress in nonalcoholic steatohepatitis. Alcohol Clin Exp Res 31: S61-S66, 2007.

23. Yang S, Zhu H, Li Y, et al: Mitochondrial adaptations to obesityrelated oxidant stress. Arch Biochem Biophys 378: 259-268, 2000.

24. Park YN, Yang CP, Fernandez GJ, Cubukcu O, Thung SN and Theise ND: Neoangiogenesis and sinusoidal 'capillarization' in dysplastic nodules of the liver. Am J Surg Pathol 22: 656-662, 1998.

25. Theuerkauf I, Zhou H and Fischer HP: Immunohistochemical patterns of human liver sinusoids under different conditions of pathologic perfusion. Virchows Arch 438: 498-504, 2001.

26. Marra F, Aleffi S, Bertolani C, Petrai I and Vizzutti F: Adipokines and liver fibrosis. Eur Rev Med Pharmacol Sci 9: 279-284, 2005.

27. Larter CZ and Farrell GC: Insulin resistance, adiponectin, cytokines in NASH: Which is the best target to treat? J Hepatol 44: 253-261, 2006.

28. Leclercq IA, Farrell GC, Schriemer R and Robertson GR: Leptin is essential for the hepatic fibrogenic response to chronic liver injury. J Hepatol 37: 206-213, 2002.
29. Musso G, Gambino R, Biroli G, et al: Hypoadiponectinemia predicts the severity of hepatic fibrosis and pancreatic Beta-cell dysfunction in nondiabetic nonobese patients with nonalcoholic steatohepatitis. Am J Gastroenterol 100: 2438-2446, 2005.

30. Kamada Y, Tamura S, Kiso S, et al: Enhanced carbon tetrachloride-induced liver fibrosis in mice lacking adiponectin. Gastroenterology 125: 1796-1807, 2003.

31. Honda H, Ikejima K, Hirose M, et al: Leptin is required for fibrogenic responses induced by thioacetamide in the murine liver. Hepatology 36: 12-21, 2002.

32. Saxena NK, Ikeda K, Rockey DC, Friedman SL and Anania FA: Leptin in hepatic fibrosis: evidence for increased collagen production in stellate cells and lean littermates of ob/ob mice. Hepatology 35: 762-771, 2002.

33. Sierra-Honigmann MR, Nath AK, Murakami C, et al: Biological action of leptin as an angiogenic factor. Science 281: 1683-1686, 1998. 